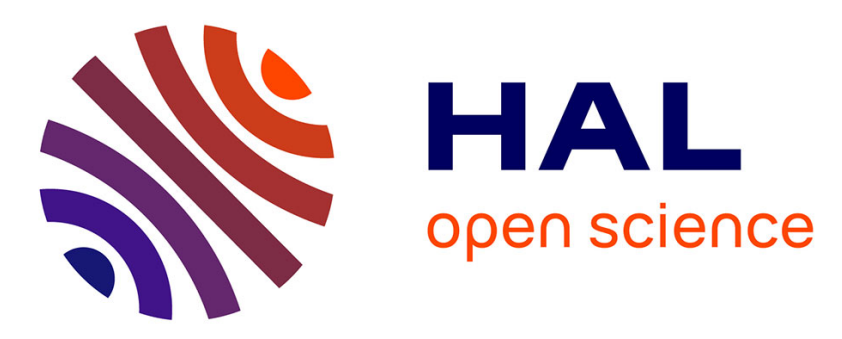

\title{
A Fourier approach to Lagrangian vortex detection
} Anass El Aouni, Hussein Yahia, Khalid Daoudi, Khalid Minaoui

\section{To cite this version:}

Anass El Aouni, Hussein Yahia, Khalid Daoudi, Khalid Minaoui. A Fourier approach to Lagrangian vortex detection. Chaos: An Interdisciplinary Journal of Nonlinear Science, 2019, 29 (9), pp.093106. 10.1063/1.5115996. hal-02280186

\section{HAL Id: hal-02280186 https://hal.inria.fr/hal-02280186}

Submitted on 6 Sep 2019

HAL is a multi-disciplinary open access archive for the deposit and dissemination of scientific research documents, whether they are published or not. The documents may come from teaching and research institutions in France or abroad, or from public or private research centers.
L'archive ouverte pluridisciplinaire HAL, est destinée au dépôt et à la diffusion de documents scientifiques de niveau recherche, publiés ou non, émanant des établissements d'enseignement et de recherche français ou étrangers, des laboratoires publics ou privés. 


\title{
A Fourier approach to Lagrangian vortex detection
}

\author{
Anass El Aouni, ${ }^{1, a)}$ Hussein Yahia, ${ }^{1}$ Khalid Daoudi, ${ }^{1}$ and Khalid Minaoui ${ }^{2}$ \\ 1) Geostat Team, INRIA Bordeaux Sud-Ouest, 33400 Talence, France \\ 2) University Mohammed V, Faculty of Sciences, LRIT, 10106 Rabat, Morocco
}

(Dated: 6 September 2019)

\begin{abstract}
We study the transport properties of coherent vortices over a finite time duration. Here we reveal that such vortices can be identified based on frequency-domain representation of Lagrangian trajectories. We use Fourier analysis to convert particles' trajectories from their time domain to a presentation in the frequency domain. We then identify and extract coherent vortices as material surfaces along which particles' trajectories share similar frequencies. Our method identifies all coherent vortices in an automatic manner, showing high vortices' monitoring capacity. We illustrate our new method by identifying and extracting Lagrangian coherent vortices in different two- and three-dimensional flows.
\end{abstract}

Keywords: Lagrangian coherent vortex, eddies, fluid dynamics, Fourier transform, frequency domain analysis.

\begin{abstract}
Various vortex definitions have been introduced in the literature together with their automatic detection. These formulations generally fall into two classes: Eulerian and Lagrangian. Here we take a very different approach to define Lagrangian coherent vortices. Our method is based on a frequency-domain representation of Lagrangian trajectories. It simply defines vortices as material surfaces along which particles' trajectories share similar frequencies.
\end{abstract}

\section{INTRODUCTION}

Lagrangian coherent structures (LCSs) are differentiated surfaces of trajectories in a dynamical system that exert a significant effect on adjacent trajectories over a time interval of interest ${ }^{1}$. These LCSs are mainly structured into three physical shapes; hyperbolic LCSs which act as repelling or attracting neighboring material elements with locally the highest rate over a given finitetime interval, parabolic LCSs which serve as generalized jet cores and elliptic LCSs serve as generalized coherent vortex boundaries in finite-time unsteady flows. In the geophysical fluid dynamics community these structures are referred to as mesoscale and submesoscale filaments, jets and eddies ${ }^{2}$.

In this paper, we are interested in physical structures of elliptical shapes. Such physical structures are omnipresent in the ocean and usually exhibit different properties to their surroundings. They are known to stir and mix surroundings water masses as well as by their ability to trap and carry fluid properties in coherent manner. In the present work, we are interested on those that live long-enough to be observed despite their environment's chaotic nature. These mesoscale eddies have an important role to play in climate change arising from their

\footnotetext{
a)Electronic mail: anass.el-aouni@inria.fr; anas.elaouni@gmail.com
}

influence on the circulation by transporting temperature and salinity. They are also known to contribute in maintaining the extra-tropical climate through the meridional transport of heat from the tropics to the poles ${ }^{3}$.

As these mesoscale eddies have a remarkable impact on the global circulation, their systematic and precise detection has been of great concern over the last two decades $^{4-7}$. Several vortex definitions have been introduced in the literature together with their automatic detection $^{8-10}$, most of these formulations are of Eulerian nature. These latter approaches make use of instantaneous velocity field to detect vortices boundaries. However, these vortices boundaries fail to coherently carry and transport their encircled water masses, instead, they stretch, deform and develop filaments ${ }^{11}$.

On the other hand, Lagrangian methods are powerful tools to identify coherent vortices because they take into account the time-evolution of particles trajectories ${ }^{6,7,11}$. Various dynamical systems approaches have been presented to define a Lagrangian transport characterization of nonautonomous chaotic dynamical systems. These approaches generally fall into different classes. Probabilistic techniques, which study the evolution of probability densities and almost-invariant sets ${ }^{5,12-16}$ and geometric approaches which use invariant manifolds. $\operatorname{In}^{6}$ the authors introduce a variational principle for coherent material vortices, where vortices' boundaries are sought as elliptic LCSs. This method has been reformulated such that it can be solved via the variational level set methodology ${ }^{17}$. These stretching-based variational methods rely on a precise computation of Cauchy-green tensor and its invariants, which requires accurate differentiation of particles trajectories with respect to their initial positions ${ }^{18}$. In ${ }^{19}$ authors propose a clustering approach to identify coherent structures by classifying fluid particles into coherent and incoherent. This was done by defining coherent structures as a group of trajectories that conserve short distances between themselves relative to the others outside the structure. This method is simple to implement and requires no differentiation of particles trajectories with respect to their initial positions but it requires a well-defined eigengap ${ }^{18}$. $\operatorname{In}^{7}$, authors use the Lagrangian- 
averaged vorticity deviation to identify Lagrangian coherent vortices. This is based on a unique decomposition of gradient deformation into product of pure strain and pure rotation gradients ${ }^{20}$. This approach is simple and low in computation cost but it requires large computational domain for spatial mean of vorticity ${ }^{18}$.

Our approach is based on frequency-domain analysis of Lagrangian particle trajectories. We represent particles' trajectory in their frequency domain in the form of sinusoid. Each sinusoid is characterized by an amplitude which gives information about vortex's diameter, and frequency which measures the vortex-turn-over time. The latter part yields an objective measure of material rotation. We define Lagrangian coherent vortex as closed material surfaces in which fluid parcels complete the same rotation. In the frequency domain, this turns out to be filled with tubular level-sets of particle trajectories' frequencies. This paper is organized as follows: Section II describes the setup and outlines the main computational tool. Section III discusses and illustrates particles trajectories in time domain. Section IV describes the representation of particles' trajectories in frequency domain. Section V presents and details our new approach. Section VI illustrate our method via different fluid simulations. Conclusion is drawn in the last Section.

\section{SET-UP}

We consider a time-dependent smooth vector field:

$$
\mathbf{v}(\mathbf{x}, t), \quad \mathbf{x} \in \mathbb{R}^{3}, \quad t \in[\alpha, \beta]
$$

and its associated ordinary differential equation:

$$
\dot{\mathbf{x}}=\mathbf{v}(\mathbf{x}, t), \quad \mathbf{x} \in \mathbb{R}^{3}, \quad t \in[\alpha, \beta]
$$

where $\mathbf{v}$ a smooth velocity field defined on a domain:

$$
\mathbf{U}(t) \subset \mathbb{R}^{3}, \quad \mathbf{U}=\bigcup_{t \in[\alpha, \beta]} \mathbf{U}(t) \subset \mathbb{R}^{3} \times[\alpha, \beta]
$$

The flow map is defined as the map that takes a particle from its initial location $\mathbf{x}_{0}$ at time $t_{0}$ to its location $\mathbf{x}_{t}$ at time $t$ :

$$
\mathbf{F}_{t_{0}}^{t}\left(\mathbf{x}_{0}\right):=\mathbf{x}\left(t, t_{0}, \mathbf{x}_{0}\right), \quad \alpha \leq t_{0} \leq t \leq \beta
$$

$\mathbf{x}\left(t, t_{0}, \mathbf{x}_{0}\right)$ denoting the trajectory of Eq.2 passing through a point $\mathbf{x}_{0}$ at time $t_{0}$.

Consider a material domain (defined by a set of fluid particles) $\mathcal{M}\left(t_{0}\right)$ advected by the flow. Its image at time $t$ can be expressed in term of the flow map as $\mathcal{M}(t)=$ $\mathbf{F}_{t_{0}}^{t}\left(\mathcal{M}\left(t_{0}\right)\right)$.

\section{METHOD}

The general outline of the proposed method is as follows. We produce particle trajectories by solving the ordinary differential equation 2. Next we apply the Fourier transform over each particle trajectory to represent them in the frequency domain. Finally, we seek coherent vortices as material surfaces along which particles' trajectories share similar frequencies' components.

\section{A. Frequency-domain analysis and Fourier transform}

Frequency-domain analysis is a cornerstone of signal and system analysis. While time-domain analysis shows how a mathematical function or a signal changes over time, frequency-domain analysis shows how their energies are distributed over a domain of frequencies. Furthermore, observing a given system from frequency point of view usually gives an intuitive interpretation of the qualitative behavior of the system. A given signal contains frequency information that can be computed using the Fourier transform which is recalled below.

If $x(t)$ is any signal, either integrable or of finite energy, the Fourier transform of $x, \mathcal{F}(x)(\xi)$, measures, at each frequency $\xi$, the amount of periodic information contained in $x$, by simply evaluating $<x \mid e^{-i \xi}>$ through an integral computation:

$$
\mathcal{F}(x)(\xi)=\int_{\mathbb{R}} x(t) e^{-i t \xi} d t
$$

with the frequency spectrum defined as the mapping $\xi \mapsto|\mathcal{F}(\xi)|^{2}$

If $x(t)$ is absolutely integrable on ] $-\infty,+\infty[$, then $\mathcal{F}(\xi)$ is continuous, $\mathcal{F}(\xi) \rightarrow 0$ as $\xi \rightarrow \pm \infty$, and

$$
|\mathcal{F}(\xi)| \leq \int_{-\infty}^{+\infty}|x(t)| d t
$$

In present scenario, continuous signals are sampled and quantized to get a discrete domain signal. This approach helps employing a variety of digital signal processing operations. In signal processing field, the Fourier transform is mostly computed using the Fast Fourier Transform algorithm (FFT).

\section{VORTEX TRAJECTORY IN TIME DOMAIN}

Various studies have addressed the problem of coherent vortex identification based on their observed trajectories. $\mathrm{In}^{21}$ authors use trajectory length to reveal the time $t_{0}$ positions of geometrical structures of different dynamics. This method is supposed to display an imprint of Lagrangian coherent structures as shown in $^{21}$. However, no 
existing mathematical link between that method's features and material coherent structures ${ }^{18}$. Moreover, the scalar field does not allow the identification and extraction of coherent vortices to allow their tracking and monitoring. Another method which make use of particles' trajectories to identify coherent structures is presented in ${ }^{19}$. This has been done by classifying fluid particles into coherent and incoherent classes. In their work, a coherent structure is described as a set of Lagrangian trajectories that keep minimum distances between themselves with respect to others outside the structure. This method is simple to implement but it has no link between material vortices and the expected spinning motion ${ }^{18}$.

Particle trajectory produced by integrating the velocity vector field in a vortex results into a loopy curve as shown in Fig.1, this figure shows trajectories' examples of particles initialized within a given vortex. Thus, a natural way to identify vortex is by locating particle trajectories which exhibit spinning motion. By neglecting the vertical components, particles' trajectories in Fig.1 result in continuous curves which intersects themselves and create closed-curves segments each time a particle returns to one of its former positions after some time such as in Fig.1. Based on this idea, authors in ${ }^{22}$ proposed a scheme to identify loops from trajectories of oceanic surface drifters. Such method indeed does identify the existence of eddies, but it does not identify vortices' cores or boundaries. Moreover this method leaves several unaddressed vortices cases where particle trajectories don't intersect themselves; for example, given a vortex with zero-translating speed and radial flow where the particles' trajectories converge according to inflow.

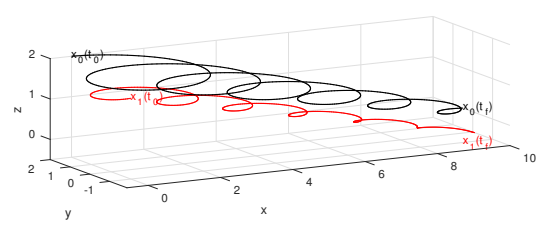

(a)

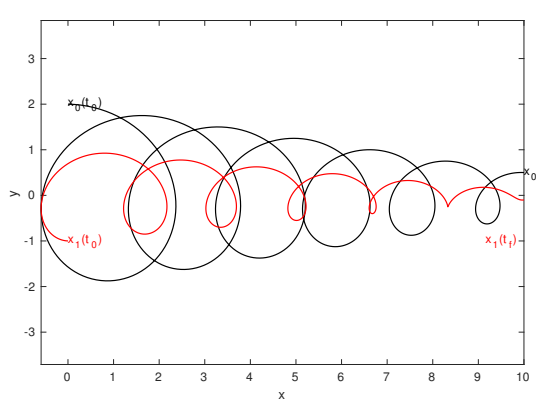

(b)

FIG. 1: a) Two particle trajectories within the same vortex: particle within the vortex boundary is presented in black while red color refers to a particle near by the vortex center. b) Their projection on the $(x, y)$ coordinates.

\section{PARTICLE TRAJECTORY IN FREQUENCY DOMAIN}

In spatial domain, particle's trajectory coordinates are represented by the Euclidean components $x, y$ and $z$, where they refer to their spatial position. In this domain, a particle trajectory within a vortex results into a loopy curve as shown in Fig.1. Its time projection over each of the spatial direction results into three components responsible for plane and vertical movement as shown in Fig.2. The $x$ and $y$ components of this particle trajectory show the emergence of an attractor associated with vortical motion. They display periodic motions characterized by a frequency presenting the vortex-turn-over time and an amplitude referring to the vortex radius.

In frequency domain, particle trajectory coordinates refer to frequencies $\xi_{x}, \xi_{y}$ and $\xi_{w}$ in each of the spatial directions. Fig. 3 shows the frequency-domain representation of particles' trajectories in the Fig.1 after excluding the drift effect. Both red and black trajectories share the same frequency but have different amplitudes. Here we see that analyzing such trajectories in their frequency domain representation is more easier than analyzing their general shapes.

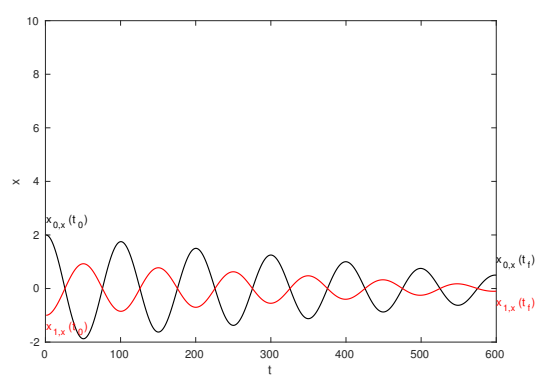

(a)

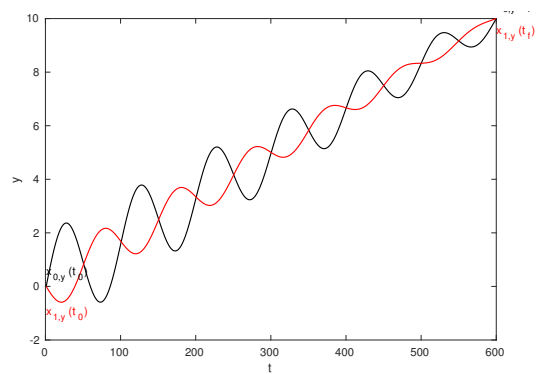

(b)

FIG. 2: Two particle trajectories within the same vortex: particle within the vortex boundary is presented in black while red color refers to a particle near by the vortex center. 


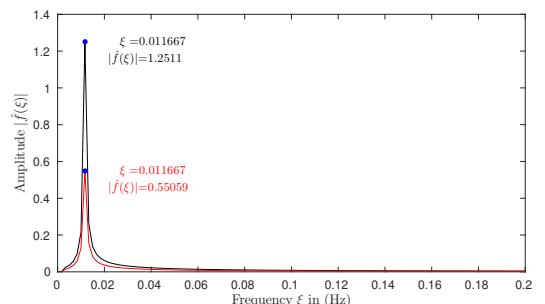

(a)

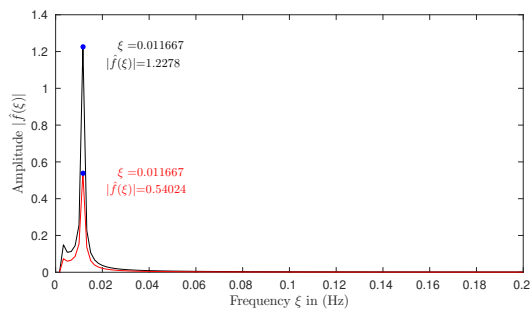

(b)

FIG. 3: Frequency-domain representation of the particles' trajectories in Fig.1, red an black trajectories have the same frequency but different amplitudes: a) within $x$-direction, b) within $y$-direction.

\section{DEFINING VORTICES FROM THEIR FREQUENCY COMPONENTS}

We seek to identify Lagrangian coherent vortices as closed material surfaces along which particles exhibit similar rotations around the same axis, and over a finite time interval. In the frequency domain representation, we seek Lagrangian coherent vortices as closed material surfaces along which particle trajectories share similar frequencies' components. In this domain, a particle trajectory can further be divided into two components; frequency which gives information about uniformly rotating flow, and the amplitude that describes the radius of a rotating particle. Based on the fact that fluid particles along vortex boundary exhibit the same polar rotation, we only consider the first part which gives information about the frequency of particle trajectory.

We define Lagrangian Trajectory Frequency $\mathcal{L} \mathcal{T} \mathcal{F}$ as:

$$
\begin{aligned}
\mathcal{L T F}^{\xi}\left(\mathbf{x}\left(\cdot, t_{0}, \mathbf{x}_{0}\right)\right) & :=\int_{t_{0}}^{t_{n}} \mathbf{x}\left(t, \mathbf{x}_{0}\right) e^{-i t \xi} d t \\
\xi & =\left(\xi_{1}, \xi_{2}, \ldots \xi_{n}\right)
\end{aligned}
$$

where $\xi=\left(\xi_{1}, \xi_{2}, \ldots \xi_{n}\right)$ presents a vector of $n$ frequencies of a given particle trajectory. With that, we are computing $n$ points of a one-dimensional Fourier transform. For a particle fluid starting from $\mathbf{x}_{0}$, the $\mathcal{L} \mathcal{T} \mathcal{F}^{\xi}\left(\mathbf{x}_{\mathbf{0}}\right)$ vector field is a dynamically consistent measure of material rotation; the average frequency weighted by the frequency spectrum:

$$
\bar{\xi}=\frac{\int_{\xi_{0}}^{\xi_{n}} \xi d \xi\left|\mathcal{L} \mathcal{T} \mathcal{F}^{\xi}\right|^{2}}{\int_{\xi_{0}}^{\xi_{n}} d \xi\left|\mathcal{L} \mathcal{T} \mathcal{F}^{\xi}\right|^{2}}
$$

gives a consistent measure of particle rotation, whereas, the mean average of the magnitude $\overline{\left|\mathcal{L T \mathcal { F }}^{\xi}\left(\mathbf{x}_{\mathbf{0}}\right)\right|}$ is a consistent measure of particle rotation radius.

We now use the $\mathcal{L} \mathcal{T} \mathcal{F}$ to identify closed material surface along which fluid parcels experience the same rotation over a time interval $\left[t_{0} t_{f}\right]$. Time $t_{0}$ positions of such material tubes are tubular level ( a convex contour in two dimensions, and a convex cylindrical or cone-shaped set in three dimensions) surfaces of the scalar $\bar{\xi}$, with $\xi \mapsto\left|\mathcal{L T F}^{\xi}\left(\mathbf{x}_{0}\right)\right|^{2}$. By definition, the $\overline{\left|\mathcal{L} \mathcal{T} \mathcal{F}^{\xi}\left(\mathbf{x}_{\mathbf{0}}\right)\right|}$ map allows the identification of vortex' center as inner most member of the vortex with the minimal rotation radius.

We summarize the $\mathcal{L} \mathcal{T} \mathcal{F}$-based vortex identification in the following definition, with its geometry illustrated in Fig.4-a.

Definition 1 For a given time interval $\left[t_{0}, t_{f}\right]$ :

- 1) Lagrangian coherent vortex is an evolving material domain $\mathbf{U}=\bigcup_{t \in\left[t_{0}, t_{f}\right]} \mathbf{U}(t) \subset \mathbb{R}^{3} \times\left[t_{0}, t_{f}\right]$ such that $\mathbf{U}\left(t_{0}\right) \subset \mathbb{R}^{3}$ is filled with a nested family of tubular level surfaces of $\bar{\xi}=\frac{\int_{\xi_{0}}^{\xi_{n}} \xi d \xi\left|\mathcal{L} \mathcal{T} \mathcal{F}^{\xi}\right|^{2}}{\int_{\xi_{0}}^{\xi_{n}} d \xi\left|\mathcal{L} \mathcal{T} \mathcal{F}^{\xi}\right|^{2}}$.

- 2) The boundary $\mathbf{B}=\bigcup_{t \in\left[t_{0}, t_{f}\right]} \mathbf{B}(t) \subset \mathbb{R}^{3} \times\left[t_{0}, t_{f}\right]$ of $\mathbf{U}$ is the outermost closed material surface of $\bar{\xi}$ in $\mathbf{U}\left(t_{0}\right)$.

In the computational world, we relax the convexity strictness for closed material surface of $\bar{\xi}$. The first reason for this convexity relaxation consists of allowing small tangential filamentation even at time $t_{0}$ of vortices' boundaries (Fig.4-b-1). The second reason consists of the nature of multi-scale data, such data shows the presence of small-scale vortices nearby the boundaries of big-scale vortices (Fig.4-b-2). The third reason consists of the representation of vortices' boundaries by discrete polygons (Fig.4-b-3). At the initial time $t_{0}$, the definition 1 identify Lagrangian coherent vortices with a simple geometry, that is by defining a parameter of maximal convexity deficiency $d_{\max }$ to allow the relaxation of convexity strictness. This enables capturing filamented parts that rotate together with the vortices without a global breakaway. The aforementioned definition allows the identification of vortex boundary $\mathbf{B}\left(\mathbf{t}_{\mathbf{0}}\right)$ which has convexity deficiency less than the maximal limit $d_{\max }$. We define the convexity deficiency of a closed curve in the plane as the ratio of the area difference between the curve and its convex hull to the area enclosed by the curve as: $d=\frac{A\left(\operatorname{Conv}\left(\mathbf{B}\left(\mathbf{t}_{\mathbf{o}}\right)\right)\right)-A\left(\mathbf{B}\left(\mathbf{t}_{\mathbf{o}}\right)\right)}{A\left(\mathbf{B}\left(\mathbf{t}_{\mathbf{o}}\right)\right)}$. Fig.4-b shows the geometrical view of the convexity deficiency parameter, the dark red color refers to the difference between the curve (in white) and its convex hull. 


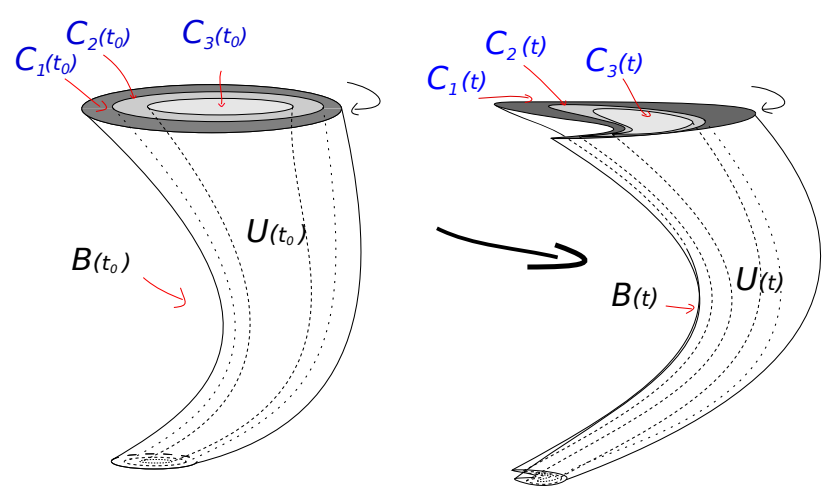

(a)

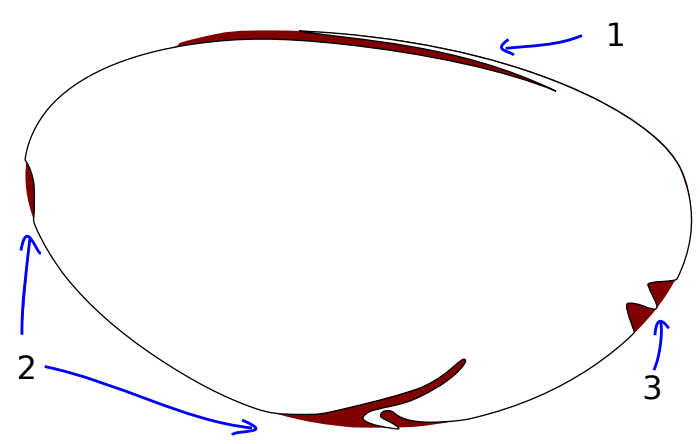

(b)

\section{EXPERIMENTS}

Here we present numerical results that confirm our theoretical predictions regarding the identification of coherent vortices.

\section{A. Two-dimensional examples}

\section{Direct numerical simulation of two-dimensional turbulence}

We solve numerically the Navier-Stokes PDE model for the time evolution of 2 components of the velocity, $u: \mathcal{D} \rightarrow \mathbb{R}^{2}$ of an incompressible fluid on a torus, $\mathcal{D}=$ $[0,2 \pi] \times[0,2 \pi]$. This can be expressed as:

$$
\begin{array}{rlrl}
\partial u_{t}+u \cdot \nabla u & =-\nabla p+\frac{1}{R e} \Delta u+f, & (x, t) & \in \mathcal{D} \times[a, b], \\
\nabla \cdot u & =0, & (x, t) \in \mathcal{D} \times[a, b], \\
\int u^{j} d x & =0, & (x, t) \in \mathcal{D} \times[a, b], j=1,2, \\
u & =u^{*}, & (x, t) \in \mathcal{D} \times\{0\}
\end{array}
$$

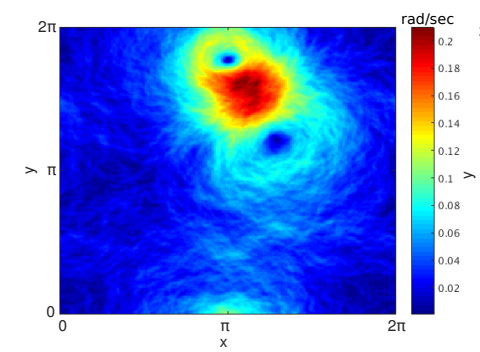

(a) $t=0$

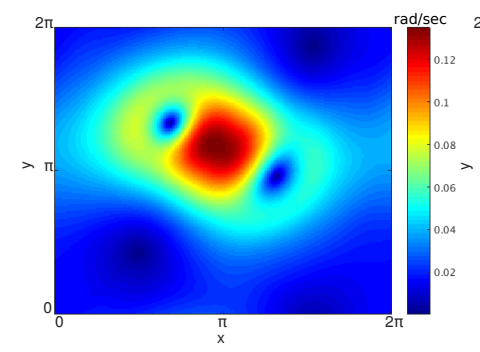

(c) $\mathrm{t}=700$

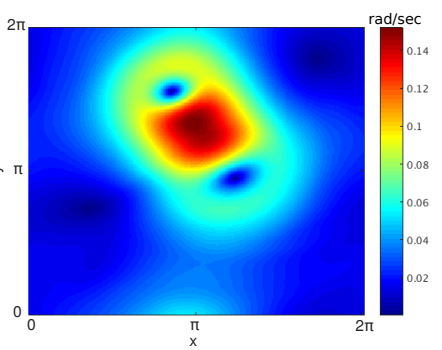

(b) $\mathrm{t}=400$

$$
\text { convexity. }
$$

Lagrangian vortices, as well as their boundaries are materials objects ${ }^{7}$. Thus, their position at a given time $t$ is only determined by Lagrangian advection:

$$
\begin{gathered}
\mathbf{U}(t)=\mathbf{F}_{t_{0}}^{t}\left(\mathbf{U}\left(t_{0}\right)\right), \quad \mathbf{B}(t)=\mathbf{F}_{t_{0}}^{t}\left(\mathbf{B}\left(t_{0}\right)\right), \\
t \in\left[t_{0}, t_{f}\right]
\end{gathered}
$$

The $\mathcal{L} \mathcal{T} \mathcal{F}$-vortex approach differs from the previous definitions, it does define vortex based on the frequency domain representation of their trajectories; more precisely, it defines vortices as closed material surfaces along which particles share similar frequencies. Our method does not require differentiation of particles trajectories with respect to their initial positions, thus, it does not require advection of high-density grids. $\mathcal{L} \mathcal{T} \mathcal{F}$-vortex might show material filament, but by definition, they will all rotate together with the vortex without breaking away. 


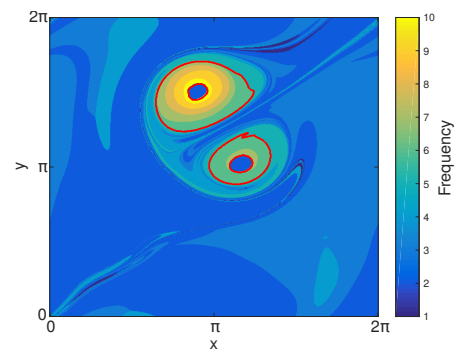

(a)

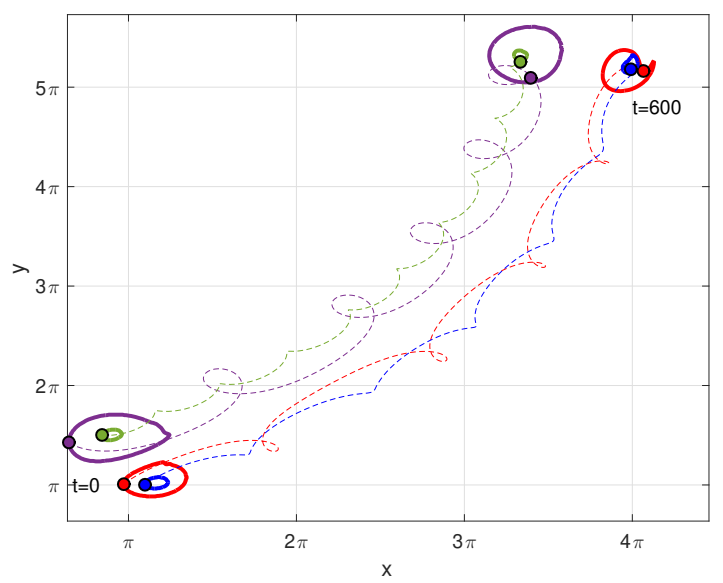

(c)

FIG. 6: a) Lagrangian coherent vortices at time $t_{0}$ extracted from the velocity field generated by Navier-Stokes model 10 using definition.1 with the $\bar{\xi}$ map shown in background, with $\xi \mapsto\left|\mathcal{L T} \mathcal{F}^{\xi}\left(\mathbf{x}_{0}\right)\right|^{2}$. b) The mean-average magnitude $\overline{\left|\mathcal{L} \mathcal{T F}^{\xi}\left(\mathbf{x}_{0}\right)\right|}$ highlighting areas of spinning movement. c) Their initial and final positions under Lagrangian advection as well as different particles initialized within their boundaries and their trajectories in Dashed line. (See the supplemental movie M1 for the complete advection sequence of these vortices.)

Where $u \cdot \nabla u$ is the inertial term which characterizes Navier-Stokes equation, and is responsible for the transfer of kinetic energy in the turbulent cascade. $\nabla p$ is the pressure gradients which guarantee the incompressibility of the flow, and $\frac{1}{R e} \Delta u$ is the the dissipative viscous term. We further assume periodic boundary conditions and use a standard pseudo-spectral method with 512 modes in each direction and $2 / 3$ dealiasing to solve the above Navier-Stokes equation with Reynolds number $R e=10^{4}$ on the time interval $t \in[0,1000]$. The model is parametrized by the pressure function $p: D \times[a, b] \rightarrow \mathbb{R}$, with no external forcing $(f=0)$. We initialize the system with the vorticity of two adjacent vortices perturbed by a random uniform distribution:

$$
\begin{aligned}
\left.\bar{\omega}\right|_{t_{0}}= & \exp \left(\frac{(x-\pi)^{2}+(y-2 \pi-\pi / 4)^{2}}{0.2}\right) \\
& -\exp \left(\frac{(x-\pi-\pi / 4)^{2}+(y-\pi-\pi / 4)^{2}}{0.8}\right)
\end{aligned}
$$

We use the vorticity stream formulation ${ }^{23}$ for implementation and get back velocity and pressure from the stream function. The flow integration is then carried out over the interval $t \in[400,1000]$, in which the turbulent flow is under fully developed turbulence, by a fourthorder Runge-Kutta method with variable step-size.

Fig.5 shows the forward simulated velocity field, obtained at four distinct time points by the Navier-Stokes model 10, over two-dimensional plane defined by: the angle of the inner and outer rings of a two dimensional torus.

To construct the $\mathcal{L T F}^{\xi}$ vector field, we consider an initial grid of $512 \times 512$ points and integrate the simulated velocity field over the time interval $\left[t_{0}=400, t_{f}=1000\right]$ using the eq.2.

Fig.6(a) shows coherent vortices extracted from the $\bar{\xi}$ map computed from the simulated realization of fluid velocity of the model 10, with $d_{\max }=10^{-3}$. For each vortex we extract the inner- and outer-most cores. Fig. 6(c) shows their final position under Lagrangian advection as well as trajectories of particles initialized on their boundaries. These Lagrangian vortices maintain their coherency, they don't stretch or fold. The complete advection sequence over the time interval $\left[t_{0}=400, t_{f}=1000\right]$ is illustrated in the movie M1.

\section{B. Two-dimensional eddies in satellite altimetry}

Here, we use sea surface velocity data to illustrate the detection of Lagrangian vortices. This velocity data is derived from satellite altimetry under the geostrophic approximation where sea-surface height $\eta(\varphi, \theta, t)$ serves as a non-canonical Hamiltonian for surface velocities in the $(\varphi, \theta)$ longitude-latitude coordinate system. The evolution of fluid particles satisfies

$$
\begin{aligned}
& \dot{\varphi}(\varphi, \theta, t)=-\frac{g}{R^{2} f(\theta) \cos \theta} \partial_{\theta} \eta(\varphi, \theta, t) \\
& \dot{\theta}(\varphi, \theta, t)=\frac{g}{R^{2} f(\theta) \cos \theta} \partial_{\varphi} \eta(\varphi, \theta, t)
\end{aligned}
$$

where $g$ is the constant of gravity, $R$ is the mean radius of the Earth and $f(\theta)=2 \Omega \sin \theta$ is the Coriolis effect, with $\Omega$ denoting the Earth's mean angular velocity. This data is produced by Ssalto/duacs multi-mission sea level products provided by AVISO (CLS/Archiving, Validation, and Interpretation of Satellite Oceanographic data $)^{24}$ with a spatial resolution of $1 / 4^{\circ}$ and temporal resolution of 7 days.

We chose the region of the Agulhas leakage in the Southern Ocean, spans from $\left[-28^{\circ} N,-4^{\circ} W\right]$ and 


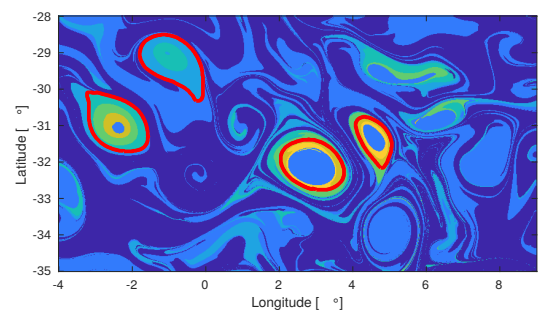

(a)

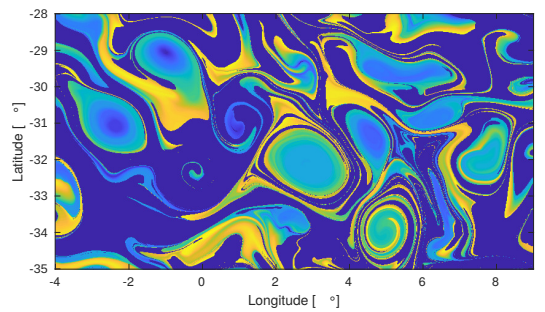

(b)

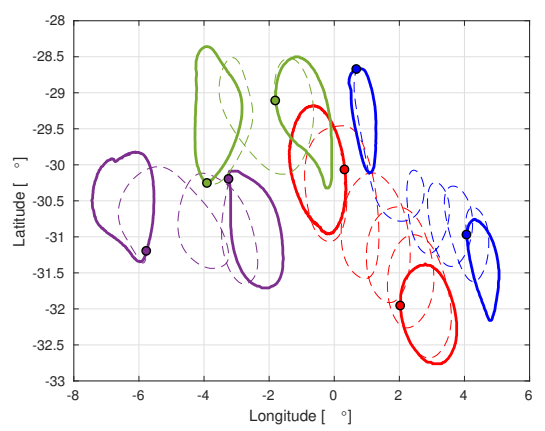

(c)

FIG. 7: a) Coherent eddies at time $t_{0}$ extracted using definition.1 with the $\bar{\xi}$ map shown in background, with $\xi \mapsto\left|\mathcal{L T}_{\mathcal{T}} \mathcal{F}^{\xi}\left(\mathbf{x}_{0}\right)\right|^{2}$. b) The mean-average magnitude $\overline{\left|\mathcal{L T F}^{\xi}\left(\mathrm{x}_{0}\right)\right|}$ highlighting areas of spinning movement. c) Their initial and final positions under Lagrangian advection as well as different particles initialized within their boundaries and their trajectories in Dashed line.

(See the supplemental movie M2 for the complete advection sequence of these vortices.)

$\left[-35^{\circ} N, 9^{\circ} \mathrm{W}\right]$. This region is well known for its longlived propagating eddies that carry water properties from the Indian ocean far into the South Atlantic ${ }^{25}$. In this study, we chose the time period between $11 / 11 / 2006$ and $11 / 1 / 2007$.

We integrate the AVISO data set (eq.12) over the period of time between $t_{0}=11$ November 2006 and $t_{f}=t_{0}+90$ days over an initial grid of particles with step size $\Delta x_{0}=1 / 50^{\circ}$. We show in Fig.7(a) $\bar{\xi}$ map computed from the satellite velocity field (eq.12). In the same image, we show coherent eddies' boundaries as they are extracted from the $\bar{\xi}$ map, with $d_{\max }=10^{-3}$. In Fig.7(c), we show their initial and final position under Lagrangian advection as well as trajectories of particles initialized on their boundaries. These eddies remain coherent. The complete advection sequence over the time interval $[0$, $90]$ is illustrated in the movie M2.

\section{Three-dimensional Agulhas eddies in a data-assimilating circulation model}

Here, we apply $\mathcal{L} \mathcal{T} \mathcal{F}$-based vortex extraction to a three-dimensional unsteady velocity field set obtained from the Southern Ocean state estimation (SOSE) model $^{26}$. The domain of the data set lies in the area of the Agulhas leakage in the Southern Ocean.

Our Lagrangian study covers a period of $T=30$ days, ranging from $t_{0}=15$ May 2006 to $t=15$ june 2006. As in $^{7}$, we select the computational domain bounded by longitudes $\left[11^{\circ} \mathrm{E}, 16^{\circ} \mathrm{E}\right]$, latitudes $\left[37^{\circ} S, 33^{\circ} \mathrm{S}\right]$ and depths $[7,2000] \mathrm{m}$. We compute the $\mathcal{L} \mathcal{T} \mathcal{F}$ vector field over a uniform grid of $150 \times 160 \times 60$ points, and identify a Lagrangian coherent eddy.

Fig.8(a) shows the three-dimensional field of the $\bar{\xi}$, $\xi \mapsto\left|\mathcal{L T F}^{\xi}\left(\mathrm{x}_{0}\right)\right|^{2}$ with the eddy's inner- and outer-most cores enclosed with red color. Particles belonging to these two cores have different frequencies. Fig.8(b) shows the initial position of a coherent Lagrangian eddy boundary (dark red), extracted as level sets of $\bar{\xi}$. Also shown is a nearby $\overline{\left|\mathcal{L} \mathcal{T F}^{\xi}\right|}$ level surface outside the eddy boundary, illustrating the complexity of the near-surface mixing region enclosing the eddy. Fig.8(c) gives a full view of the Lagrangian eddy. Fig.8(d) shows the initial and the materially advected position of the eddy at the final time $t_{f}=t_{0}+30$ days, whereas, Fig.8(e) shows the initial and final position of different eddy's layers presented in different colors. As anticipated there is moderate tangential filamentation in the material eddy boundary, but precisely no breakaway from the rotating eddy core. This high degree of material coherence in the presence of the complex surrounding material mixing demonstrates the efficiency of $\mathcal{L} \mathcal{T} \mathcal{F}$-based vortex detection.

\section{CONCLUSION}

We have presented an objective frequency-domain definition of Lagrangian coherent vortices as closed material surfaces along which fluid parcels exhibit the same intrinsic rotation. This coherent material is obtained from the frequency-domain representation of their trajectories. This intrinsic rotating material is expressible as material tube characterized by a given frequency. We illustrated our new method on different two- and three-dimensional flows. Our results show that vortices boundaries obtained are sharply defined. Our approach does not require advection of high-density material grids, a generally an expensive computational procedure in Lagrangian coherence calculations. Moreover, it is suitable to applications to float data. 


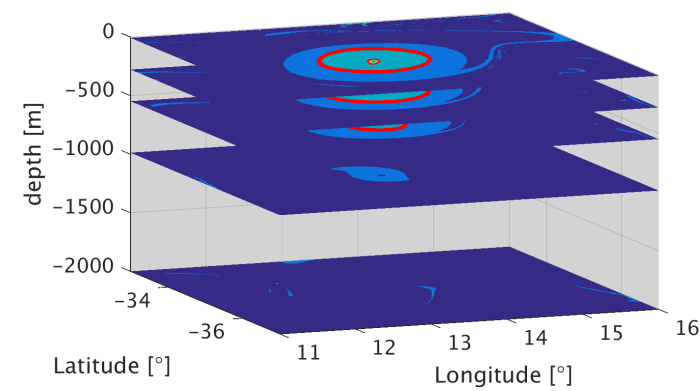

(a)

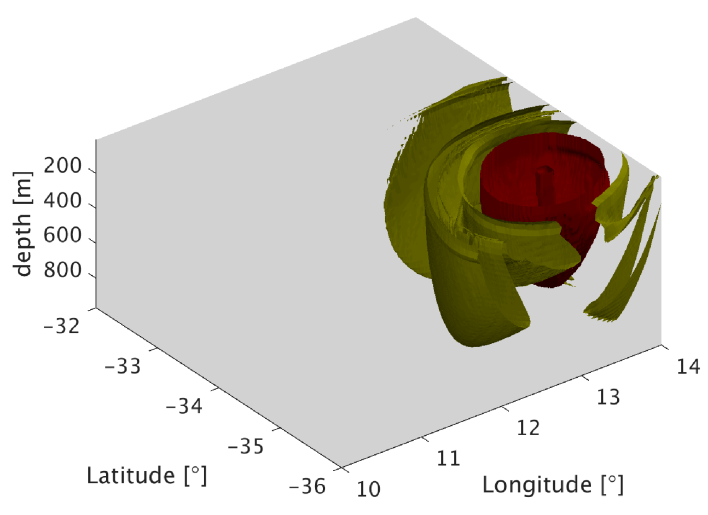

(b)

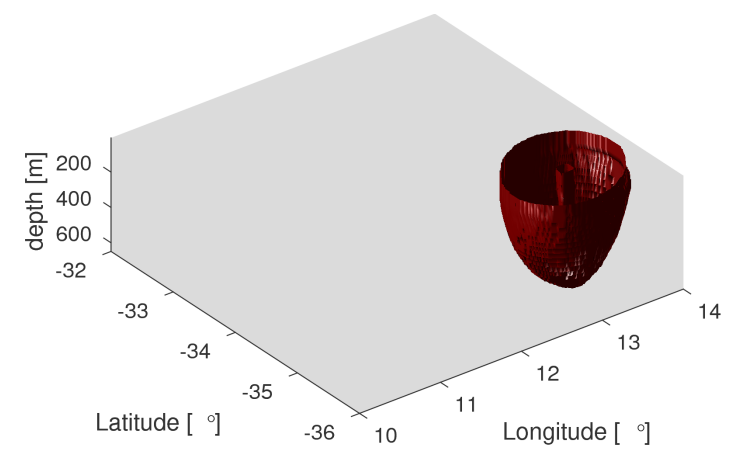

(c)

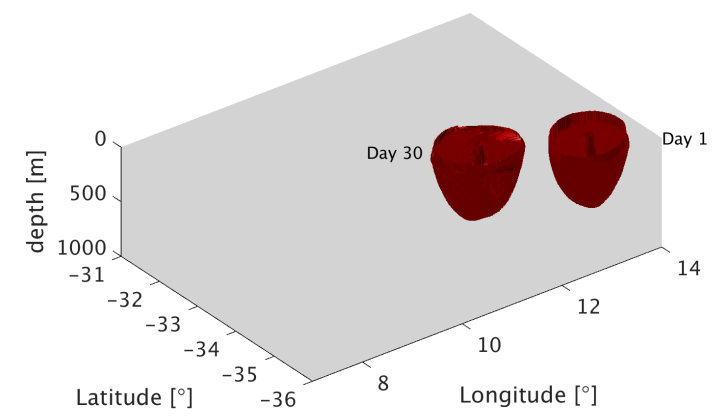

(d)

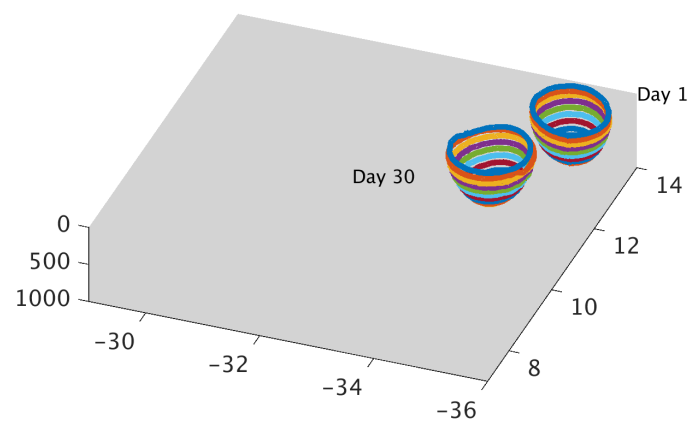

(e)

FIG. 8: a) A three-dimensional field of the $\bar{\xi}$, with $\xi \mapsto\left|\mathcal{L T F}^{\xi}\left(\mathbf{x}_{0}\right)\right|^{2}$. b) Representative level surfaces of $\bar{\xi}$. The dark red surface is extracted using the definition.1, marking the vortex boundary for a mesoscale coherent Lagrangian eddy, extending from $7 \mathrm{~m}$ down to $600 \mathrm{~m}$ in depth. The green surface is a nearby level surface of $\overline{\left|\mathcal{L} \mathcal{T F}^{\xi}\right|}$ outside the

Lagrangian eddy region. c) Full view of the Lagrangian eddy boundary. d) The initial and the advected eddy boundary 30 days later, whereas e) shows initial and the advected images of different layers of the eddy. (See the supplemental movie M3 and M4 for the complete advection sequence of these vortices.)

\section{SUPPLEMENTARY MATERIAL}

See the supplemental movie M1 for the complete advection sequence of the vortices in Fig. 6.

See the supplemental movie M2 for the complete advection sequence of the vortices in Fig.7.

See the supplemental movie M3 and M4 for the complete advection sequence of the vortices in Fig. 8.

\section{ACKNOWLEDGMENTS}

This work was supported by the French-Moroccan PHC-Toubkal project $n^{\circ}$ TBK/16-24 and PPR2-6 project. A. El Aouni is partially supported by the French project BOOSTE TON DOC 2019. 
${ }^{1}$ G. Haller and G. Yuan, "Lagrangian coherent structures and mixing in two-dimensional turbulence," Physica D: Nonlinear Phenomena 147, 352-370 (2000).

${ }^{2} \mathrm{~T}$. Peacock and J. Dabiri, "Introduction to focus issue: Lagrangian coherent structures," Chaos: An Interdisciplinary Journal of Nonlinear Science 20, 017501 (2010), https://doi.org/10.1063/1.3278173.

${ }^{3}$ R. Farneti, T. L. Delworth, A. J. Rosati, S. M. Griffies, and F. Zeng, "The role of mesoscale eddies in the rectification of the southern ocean response to climate change," Journal of Physical Oceanography 40, 1539-1557 (2010).

${ }^{4}$ D. Karrasch, F. Huhn, and G. Haller, "Automated detection of coherent lagrangian vortices in two-dimensional unsteady flows," in Proc. R. Soc. A, Vol. 471 (The Royal Society, 2015) p. 20140639.

${ }^{5}$ G. Froyland, N. Santitissadeekorn, and A. Monahan, "Transport in time-dependent dynamical systems: Finite-time coherent sets," Chaos: An Interdisciplinary Journal of Nonlinear Science 20, 043116 (2010).

${ }^{6}$ G. Haller and F. J. Beron-Vera, "Coherent lagrangian vortices: The black holes of turbulence," Journal of Fluid Mechanics 731 (2013).

${ }^{7}$ G. Haller, A. Hadjighasem, M. Farazmand, and F. Huhn, "Defining coherent vortices objectively from the vorticity," Journal of Fluid Mechanics 795, 136-173 (2016).

${ }^{8}$ L. Zhang, Q. Deng, R. Machiraju, A. Rangarajan, D. Thompson, D. K. Walters, and H.-W. Shen, "Boosting techniques for physics-based vortex detection," in Computer Graphics Forum, Vol. 33 (Wiley Online Library, 2014) pp. 282-293.

${ }^{9}$ J. Sahner, T. Weinkauf, N. Teuber, and H.-C. Hege, "Vortex and strain skeletons in eulerian and lagrangian frames," IEEE Transactions on Visualization and Computer Graphics 13 (2007).

${ }^{10} \mathrm{M}$. Herrmann, "A eulerian level set/vortex sheet method for twophase interface dynamics," Journal of Computational Physics 203, 539-571 (2005).

${ }^{11} \mathrm{G}$. Haller, "An objective definition of a vortex," Journal of fluid mechanics 525, 1-26 (2005).

${ }^{12} \mathrm{G}$. Froyland, "An analytic framework for identifying finite-time coherent sets in time-dependent dynamical systems," Physica D: Nonlinear Phenomena 250, 1-19 (2013).

${ }^{13}$ G. Froyland, C. Horenkamp, V. Rossi, N. Santitissadeekorn, and A. S. Gupta, "Three-dimensional characterization and tracking of an agulhas ring," Ocean Modelling 52, 69-75 (2012).

${ }^{14}$ W. R. Crawford, P. J. Brickley, and A. C. Thomas, "Mesoscale eddies dominate surface phytoplankton in northern gulf of alaska," Progress in Oceanography 75, 287-303 (2007).

${ }^{15}$ A. Sobel, R. Plumb, and D. Waugh, "Methods of calculating transport across the polar vortex edge," Journal of the atmospheric sciences 54, 2241-2260 (1997).

${ }^{16}$ E. R. Nash, P. A. Newman, J. E. Rosenfield, and M. R. Schoeberl, "An objective determination of the polar vortex using ertel's potential vorticity," Journal of Geophysical Research: Atmospheres 101, 9471-9478 (1996).

${ }^{17}$ A. Hadjighasem and G. Haller, "Level set formulation of twodimensional lagrangian vortex detection methods," Chaos: An Interdisciplinary Journal of Nonlinear Science 26, 103102 (2016).

${ }^{18}$ A. Hadjighasem, M. Farazmand, D. Blazevski, G. Froyland, and G. Haller, "A critical comparison of lagrangian methods for coherent structure detection," Chaos: An Interdisciplinary Journal of Nonlinear Science 27, 053104 (2017).

${ }^{19}$ A. Hadjighasem, D. Karrasch, H. Teramoto, and G. Haller, "Spectral-clustering approach to lagrangian vortex detection," Physical Review E 93, 063107 (2016).

${ }^{20} \mathrm{G}$. Haller, "Dynamic rotation and stretch tensors from a dynamic polar decomposition," Journal of the Mechanics and Physics of Solids 86, 70-93 (2016).

${ }^{21}$ A. M. Mancho, S. Wiggins, J. Curbelo, and C. Mendoza, "Lagrangian descriptors: A method for revealing phase space structures of general time dependent dynamical systems," Communications in Nonlinear Science and Numerical Simulation 18, 3530-
$3557(2013)$

${ }^{22}$ C. Dong, Y. Liu, R. Lumpkin, M. Lankhorst, D. Chen, J. C. McWilliams, and Y. Guan, "A scheme to identify loops from trajectories of oceanic surface drifters: an application in the kuroshio extension region," Journal of Atmospheric and Oceanic Technology 28, 1167-1176 (2011).

${ }^{23}$ W. Spotz and G. Carey, "High-order compact scheme for the steady stream-function vorticity equations," International Journal for Numerical Methods in Engineering 38, 3497-3512 (1995).

${ }^{24} \mathrm{Http}: / /$ www.aviso.oceanobs.com/duacs/.

${ }^{25}$ W. d. Ruijter, A. Biastoch, S. Drijfhout, J. Lutjeharms, R. Matano, T. Pichevin, P. v. Leeuwen, and W. Weijer, "Indianatlantic interocean exchange: Dynamics, estimation and impact," Journal of Geophysical Research: Oceans 104, 2088520910 (1999).

${ }^{26}$ M. R. Mazloff, P. Heimbach, and C. Wunsch, "An eddypermitting southern ocean state estimate," Journal of Physical Oceanography 40, 880-899 (2010). 